\title{
Successful Treatment with S-1 and Oxaliplatin Combination Therapy in an Elderly Patient with Metastatic Colorectal Cancer Initially Presenting with Membranous Nephropathy
}

\author{
Mototsugu Matsunaga ${ }^{a} \quad K^{2}$ eisuke Miwa ${ }^{a}$ Yosuke Oka ${ }^{b}$ \\ Tomoyuki Ushijima $a^{a, c} \quad K^{a}$ otaro Yuge ${ }^{b}$ Masaru Fukahori ${ }^{a, c}$ \\ Mitsuhiro Katagiri, ${ }^{a, b}$ Yoshito Akagi \\ ${ }^{a}$ Multidisciplinary Treatment Cancer Center, Kurume University Hospital, ${ }^{\mathrm{b}}$ Department of \\ Surgery, and 'Division of Gastroenterology, Department of Medicine, Kurume University \\ School of Medicine, Kurume, Japan
}

\section{Key Words}

Membranous nephropathy $\cdot$ S- 1 and oxaliplatin $\cdot$ Colorectal cancer

\begin{abstract}
The incidence, morbidity, and mortality of colorectal cancer are increasing, largely owing to an increasingly aging population. Additionally, along with the increasing age of cancer patients, the number of patients with various comorbidities such as membranous nephropathy is also rising, and problems associated with the administration of chemotherapy to elderly patients with these conditions are becoming more common. Herein, we describe a case involving an 80-year-old woman who presented with general malaise, edematous limbs, and pleural effusion. An abdominal CT revealed multiple, relatively large, metastatic lesions in a wide area of the liver and left pleural effusion, and she was accordingly diagnosed with membranous nephropathy secondary to ascending colon cancer and multiple liver metastases. Despite her advanced age and the presence of membranous nephropathy, her general condition was favorable and chemotherapy was hence administered. Taking the toxicity profiles and the patient's preference into consideration, S-1 and oxaliplatin (SOX) therapy was selected, which showed a good tolerability. An abdominal CT after 8 cycles of SOX therapy revealed a marked reduction in the metastatic lesions in the liver and a decrease in the left
\end{abstract}

KARGER 125is $\quad \begin{aligned} & \text { Dr. Mototsugu Matsunaga } \\ & \text { Multidisciplinary Treatment Cancer Center } \\ & \text { Kurume University Hospital } \\ & 67 \text { Asahimachi, Kurume, Fukuoka, 830-0011 (Japan) } \\ & \text { E-Mail mmandmacgyver@yahoo.co.jp }\end{aligned}$


Matsunaga et al.: Successful Treatment with S-1 and Oxaliplatin Combination Therapy in an Elderly Patient with Metastatic Colorectal Cancer Initially Presenting with Membranous Nephropathy

pleural effusion, and the levels of tumor markers also decreased (partial response). At the latest follow-up, after the completion of 16 cycles, the condition of the patient remained stable, without any apparent signs of progressive disease. Based on this case, we conclude that, even for elderly patients with systemic complications or comorbid diseases, standard treatments should be considered after their general conditions, and therapeutic regimens have been sufficiently examined.

(c) 2015 S. Karger AG, Basel

\section{Introduction}

The morbidity and mortality rates of colorectal cancer are increasing. In cases of progressive and recurrent colorectal cancer ineligible for radical resection, although the survival time has been prolonged because of the advent of various drugs, a cure is difficult to achieve, and the prognosis of colorectal cancer patients remains poor. In addition, with the increasingly aging population, the number of cancer patients with various comorbidities, such as cerebrovascular disorders and hepatic/renal dysfunction, is also increasing; consequently, problems associated with the administration of chemotherapy to elderly patients with these conditions are also becoming more common. However, even for these patients, the standard treatment should be considered after their general condition, and therapeutic regimens have been sufficiently examined. In fact, it seems that many patients, including elderly patients with colorectal cancer complicated by membranous nephropathy, such as the patient described herein, can be safely treated by adding sufficient supportive therapy.

We experienced a case involving an elderly patient with metastatic colorectal cancer that was detected at the onset of membranous nephropathy and that responded to S-1 and oxaliplatin combination (SOX) therapy. Moreover, we also conducted a brief literature review based on this case.

\section{Case Report}

The patient was an 80-year-old woman. She presented with general malaise, edematous limbs, and pleural effusion, and was suspected to have a nephrotic syndrome. Accordingly, she was referred to our hospital from her local clinic. At her initial visit, the test results showed a total protein level of $5.03 \mathrm{~g} / \mathrm{dl}$, albumin level of $2.36 \mathrm{~g} / \mathrm{dl}$, a total cholesterol level of $265 \mathrm{mg} / \mathrm{dl}$, a strong positivity for urine protein and a daily urine protein excretion of $1.1 \mathrm{~g}$. After undergoing various detailed examinations at the Department of Nephrology, she was diagnosed with membranous nephropathy secondary to ascending colon cancer and multiple liver metastases. For the colonic stricture and bleeding, a right hemicolectomy and ileostomy were first performed. After surgery, she was referred to our department for chemotherapy. Despite her advanced age and the presence of membranous nephropathy, no bone marrow, hepatic or renal dysfunction was noted, and her general condition was favorable, with a performance status 1 . Thus, chemotherapy was administered after informed consent had been obtained from the patient. Bevacizumab was not administered owing to proteinuria caused by the membranous nephropathy, and anti-epidermal growth factor receptor drugs could not be administered owing to the presence of a KRAS G12V mutation. Considering the toxicity profiles and the patient's preference, SOX therapy was selected as the primary treatment [1]. The SOX regimen consisted of S-1 (40-60 mg depending on the patient's body surface area) given orally twice daily for 2 consecutive weeks and $100 \mathrm{mg} / \mathrm{m}^{2}$ 
Matsunaga et al.: Successful Treatment with S-1 and Oxaliplatin Combination Therapy in an Elderly Patient with Metastatic Colorectal Cancer Initially Presenting with Membranous Nephropathy

oxaliplatin (initiated at a dose reduced by 1 step in consideration of her age and underlying diseases) administered intravenously on day 1, followed by a 1-week rest period to comprise a 3-week cycle. On day 3 of the first cycle of SOX therapy, high fever, shivering, and anorexia (grade 3) were observed. These symptoms were determined to have resulted from infection along with the metastatic lesions in the liver. Consequently, the oral administration of S-1 was suspended, and antibiotic therapy was initiated. The symptoms were relieved by conservative therapy, and the oral administration of S-1 was resumed on day 10 . The course after the resumption of the oral administration of S-1 was uneventful, without any particular problems. Because of the adverse events, the dose of oxaliplatin was further reduced (75 $\mathrm{mg} / \mathrm{m}^{2}$ ) in the second cycle to allow for a treatment continuation. In the subsequent cycles, although grade 1-2 peripheral sensory neuropathy caused by oxaliplatin was observed, the tolerability of the treatment was considered good.

An abdominal CT performed before the initiation of chemotherapy revealed multiple, relatively large, metastatic lesions in a wide area of the liver as well as left pleural effusion (fig. 1). Another abdominal CT scan at the point of maximum treatment effect (after the completion of 8 cycles of SOX therapy) revealed a marked reduction in the metastatic lesions in the liver and a decrease in the left pleural effusion (fig. 2). In accordance with the improved imaging findings, the levels of tumor markers also decreased at this time. The levels of carcinoembryonic antigen and carbohydrate antigen $19-9$ were $467.6 \mathrm{ng} / \mathrm{ml}$ and 1,881.0 $\mathrm{U} / \mathrm{ml}$, respectively, immediately before the initiation of chemotherapy; at the completion of 8 cycles of SOX therapy, they were reduced to $8.0 \mathrm{ng} / \mathrm{ml}$ and $22.7 \mathrm{U} / \mathrm{ml}$, respectively. The treatment response was classified as a partial response. According to the evaluation of the CT images at the completion of 16 cycles, the condition of the patient remains stable, without any apparent sign of progressive disease.

\section{Discussion}

Membranous nephropathy is known to occur secondary to malignancy [2]. Lefaucheur et al. [3] and Bjorneklett et al. [4] found that 7.5-10\% of patients diagnosed with membranous nephropathy have concurrent malignancies. The occurrence of membranous nephropathy has been reported in lung [5], gastric [6], and colorectal cancers [7] (among others), and Leeaphorn et al. [8] reported that colorectal cancer accounted for approximately $11 \%$ of cases of malignancies with concurrent membranous nephropathy, making it the fourth most common malignancy following lung cancer, prostate cancer, and hematologic malignancies. Although the exact incidence rate of membranous nephropathy in patients with colorectal cancer remains unknown, a report of an aggregate analysis performed in Japan in 2012 [9] showed that only $8(1.0 \%)$ of 813 cases of membranous nephropathy were associated with malignancy, and that none of these cases were colorectal cancer. Thus, the incidence rate of membranous nephropathy is assumed not to be particularly high in patients with colorectal cancer in Japan. In patients with malignancy, it is considered that membranous nephropathy is due to tumor-associated antigens and their antibodies forming immune complexes, which deposit on the glomerular basement membrane [7]. In this case, although we initiated treatment for malignancy with an expectation that this treatment would contribute to relief of the membranous nephropathy, as reported in a previous study [10], the hematological and urinary findings showed only mild relief. However, there was little evidence of deterioration of renal function or of a trend of fluid retention such as edema. 
Matsunaga et al.: Successful Treatment with S-1 and Oxaliplatin Combination Therapy in an Elderly Patient with Metastatic Colorectal Cancer Initially Presenting with Membranous Nephropathy

At present, oxaliplatin- [11] or irinotecan-based [12] therapy, combined with molecular target drugs such as bevacizumab [13], cetuximab [14] and panitumumab [15], is widely and commonly applied to unresectable metastatic colorectal cancer. In our case, owing to concerns over diarrhea caused by irinotecan and the management of an ileal stoma, we considered oxaliplatin-based therapy combined with molecular target drugs as the primary treatment. However, bevacizumab was not administered owing to proteinuria caused by the membranous nephropathy, and, furthermore, anti-epidermal growth factor receptor drugs could not be used due to the presence of a KRAS mutation. Although 5-fluorouracil/folinic acid plus oxaliplatin [11] and capecitabine plus oxaliplatin therapy [11] could also be considered valid treatment options, SOX therapy was selected for our patient in consideration of her request for a treatment that was associated with a lower toxicity profile, allowed longer intervals between visits, was mainly composed of oral medications at low doses and required shorter durations of infusion. Although infection and grade 3 anorexia were observed on day 3 of the first cycle of SOX therapy, no serious adverse event was subsequently observed. After oxaliplatin was resumed at a reduced dose, its tolerability was good.

Currently, approximately 1 year after the initiation of treatment, the status of the patient remains at partial response and shows no aggravation. In patients receiving SOX therapy combined with bevacizumab as the primary treatment for unresectable metastatic colorectal cancer, it is reported that the response rate is $62.5 \%$, and that the progression-free survival is 11.7 months [1]. Despite our patient's advanced age, an adequate treatment response was achieved with this treatment, and we plan to continue SOX therapy unless she develops progressive disease or intolerable adverse events.

In conclusion, even elderly patients with metastatic colorectal cancer and concurrent membranous nephropathy may respond to SOX therapy administered as the primary treatment. Our case suggests that, if the organ functions and general condition permit, aggressive drug therapy should be considered.

\section{Disclosure Statement}

The authors have no conflicts of interest to declare.

\section{References}

-1 Yamada Y, Takahari D, Matsumoto H, et al: Leucovorin, fluorouracil, and oxaliplatin plus bevacizumab versus S-1 and oxaliplatin plus bevacizumab in patients with metastatic colorectal cancer (SOFT): an openlabel, non-inferiority, randomized phase 3 trial. Lancet Oncol 2013;14:1278-1286.

2 Lee JC, Yamauchi H, Hopper J: The association of cancer and the nephrotic syndrome. Ann Intern Med 1966;64:41-51.

-3 Lefaucheur C, Stengel B, Nochy D, et al; GN-PROGRESS Study Group: Membranous nephropathy and cancer: epidemiologic evidence and determinants of high-risk cancer association. Kidney Int 2006;70:1510-1510.

4 Bjorneklett R, Vikse BE, Svarstad E, et al: Long-term risk of cancer in membranous nephropathy patients. Am J Kidney Dis 2007;50:396-403.

-5 Eagan JW, Lewis EJ: Glomerulopathies of neoplasia. Kidney Int 1977;11:297-306.

-6 Wakashin M, Wakashin Y, Iesato K, et al: Association of gastric cancer and nephrotic syndrome. An immunologic study in three patients. Gastroenterology 1980;78:749-756.

7 Goldstein D, Kelly R, Ralston M, et al: Chemotherapy influencing the course of nephrotic syndrome in colonic carcinoma. Med Oncol Tumor Pharmacother 1987;4:101-105.

$>8$ Leeaphorn N, Kue-A-Pai P, Thamcharoen N, et al: Prevalence of cancer in membranous nephropathy: a systematic review and meta-analysis of observational studies. Am J Nephrol 2014;40:29-35.

-9 Yokoyama H, Taguchi T, Sugiyama H, Sato H: Membranous nephropathy in Japan: analysis of the Japan Renal Biopsy Registry (J-RBR). Clin Exp Nephrol 2012;16:557-563. 


\section{Case Reports in Oncology}

\begin{tabular}{l|l}
\hline \multicolumn{2}{l}{ Case Rep Oncol 2015;8:212-216 } \\
\hline DOI: 10.1159/000382119 & $\begin{array}{l}\text { @ 2015 S. Karger AG, Basel } \\
\text { www.karger.com/cro }\end{array}$ \\
\hline
\end{tabular}

Matsunaga et al.: Successful Treatment with S-1 and Oxaliplatin Combination Therapy in an Elderly Patient with Metastatic Colorectal Cancer Initially Presenting with Membranous Nephropathy

10 Burstein DM, Korbet SM, Schwartz MM: Membranous glomerulonephritis and malignancy. Am J Kidney Dis 1993;22:5-10.

11 Cassidy J, Clarke S, Diaz-Rubio E, et al: Randomized phase III study of capecitabine plus oxaliplatin compared with fluorouracil/folinic acid plus oxaliplatin as first-line therapy for metastatic colorectal cancer. J Clin Oncol 2008;26:2006-2012.

$\$ 12$ Tournigand C, Andre T, Achille E, et al: FOLFIRI followed by FOLFOX6 or the reverse sequence in advanced colorectal cancer: a randomized GERCOR study. J Clin Oncol 2004;22:229-237.

13 Hurwitz H, Fehrenbacher L, Novotny W, et al: Bevacizumab plus irinotecan, fluorouracil, and leucovorin for metastatic colorectal cancer. N Engl J Med 2004;350:2335-2342.

14 Van Cutsem E, Kohne CH, Hitre E, et al: Cetuximab and chemotherapy as initial treatment for metastatic colorectal cancer. N Engl J Med 2009;360:1408-1417.

15 Douillard JY, Siena S, Cassidy J, et al: Final results from PRIME: randomized phase III study of panitumumab with FOLFOX4 for first-line treatment of metastatic colorectal cancer. Ann Oncol 2014;25:1346-1355.
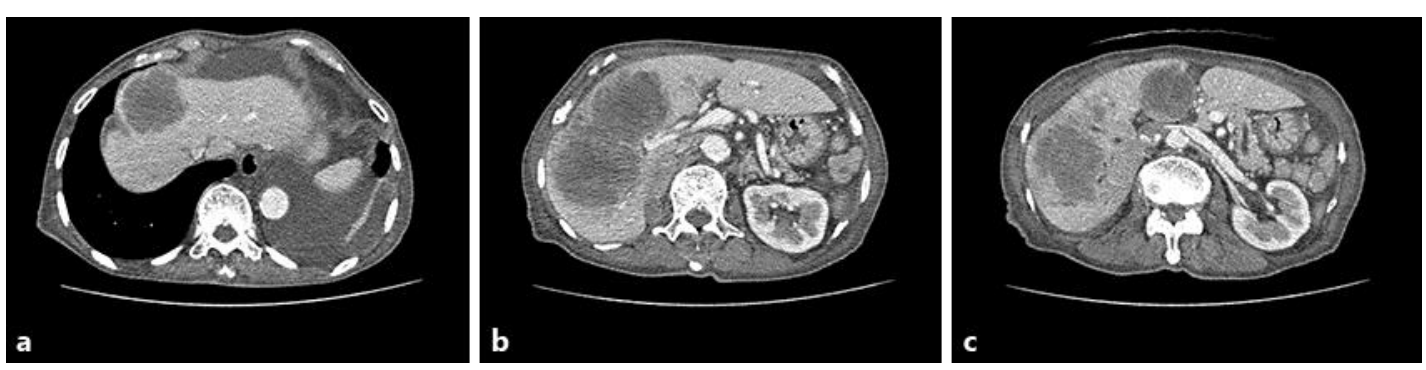

Fig. 1. a-c Abdominal CT findings before the initiation of chemotherapy. Multiple, relatively large, metastatic lesions are observed in a wide area of the liver. Left pleural effusion is also noted.
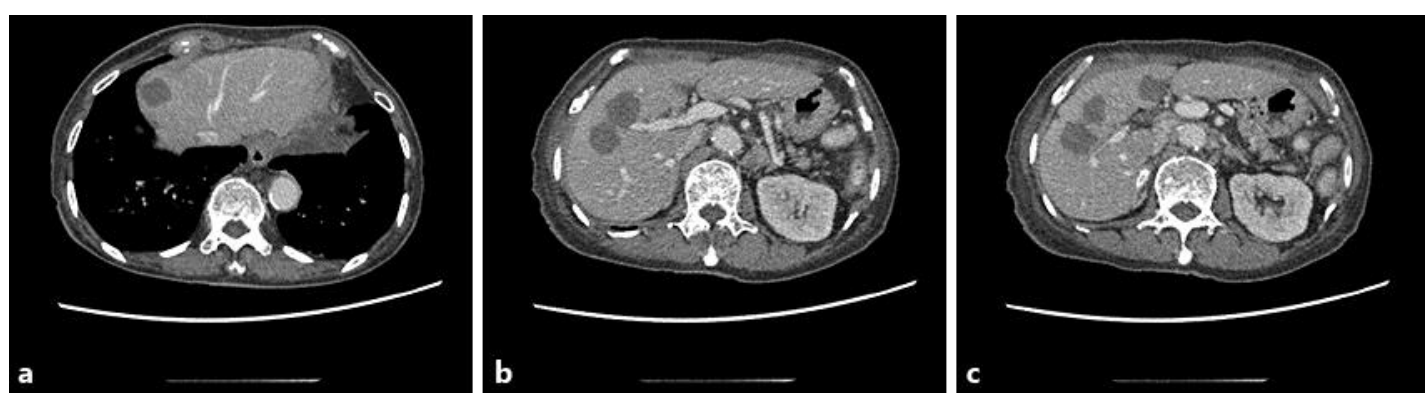

Fig. 2. a-c Abdominal CT findings at the point of maximum treatment effect (after the completion of 8 cycles of SOX therapy). A marked reduction in the metastatic lesions in the liver is observed, and the treatment response was classified as a partial response. Moreover, a decrease in the left pleural effusion is also observed. 